\title{
Microstructure and Chemical Composition Analysis of Additive Manufactured Ni-Mn-Ga Parts Sintered in Different Conditions
}

\author{
Matthew Caputo ${ }^{1}$, C. Virgil Solomon ${ }^{2}$ \\ ${ }^{1}$ Materials Science \& Engineering Program, Youngstown State University, Youngstown, Ohio, USA \\ ${ }^{2}$ Mechanical \& Industrial Engineering Department, Youngstown State University, Youngstown, Ohio, \\ USA
}

Near-stoichiometric $\mathrm{Ni}_{2} \mathrm{MnGa}$ ferromagnetic shape memory alloys (FSMA) are known for producing a large magnetic field induced strain (MFIS's) in the presence of external magnetic fields. The MFIS's are in the order of $10 \%$ for single crystals [1] and $0.5 \%$ for polycrystalline [2]. Introducing porosity into polycrystalline Ni-Mn-Ga has shown an increase in MFIS's, resulting in a stable 4.5-5.1\% MFIS [3]. These large MFIS's have attracted scientific and engineering interest, albeit the production and manufacturing of Ni-Mn-Ga is challenging due to the brittle mechanical properties, as well as the necessity to maintain a rigorous stoichiometry. Based on these recent discoveries, as well as current interest in advanced manufacturing, specifically additive manufacturing (AM), the capability to produce $\mathrm{Ni}-\mathrm{Mn}$-Ga porous parts of a desired geometry is presented. AM via binder jetting repeats the adhesion of metallic powder layers based on a CAD design, resulting in a porous near net shaped part. The purpose of this work is to examine material properties including microstructure, porosity, chemical composition, and crystallography of AM Ni-Mn-Ga parts which underwent different sintering conditions.

High purity elements of nickel (99.9 at\%), manganese (99.9 at\%), and gallium (99.9 at\%) were arc melted to produce $\mathrm{Ni}_{49.73} \mathrm{Mn}_{29.03} \mathrm{Ga}_{21.24}$ alloy. The ingots were re-melted several times and heat treated at $1273 \mathrm{~K}$ for 24 hours to ensure a homogenous metallic mixture. The ingots were then ball milled to produce powder, later used for the binder jetting AM process [4]. Fig. 1a shows a SE micrograph of prealloyed Ni-Mn-Ga ball milled powders. The powders were used to print cylindrical shaped components, Fig. 1b, sintered at $1353 \mathrm{~K}$ for 10 (Fig. 2a), 20, 30 (Fig. 2b), 40 and 50 (Fig. 2c) hours. Part sintering was done in encapsulated quartz, purged with high purity argon. Post sintering, the microstructure and chemical composition was examined using a JEOL JSM-7600F FESEM, equipped with EDAX Octane XEDS silicon drift detector. The microstructure of the AM Ni-Mn-Ga parts clearly display evidence of sintering, Fig. 1c, due to the aggregation of Ni-Mn-Ga powders.

Figures 2a to 2c shows SE micrographs of polished cross-sectioned carbon coated Ni-Mn-Ga parts sintered at 10, 30, and 50 hours, respectively. It can easily be observed that the specimen density increased with sintering time, as indicated by the reduction of pores size and distribution. Density measurements based on mass and volume, show parts sintered for 10 hours are approximately $75.9 \%$ dense, while parts sintered for 50 hours are approximately $85.0 \%$ dense. These measurements indicate the density of Ni-Mn-Ga AM parts can be controlled, based on sintering time. Chemical composition, and related crystalline structure, directly affects the functional properties of Ni-Mn-Ga FSMA's. Chemical composition of sintered materials was investigated by quantitative XEDS analysis, using a homogeneous $\mathrm{Ni}_{50.90} \mathrm{Mn}_{29.23} \mathrm{Ga}_{19.87}$ (at\%) single crystal reference sample. A summary of the chemical composition analysis is shown in Table 1. Ball milled powders appear to have more nickel content compared to sintered Ni-Mn-Ga, implying a loss of nickel during sintering. Composition for sintered samples remained fairly constant. Fig. 3 shows a summary of the XRD results (Bruker Prospector diffractometer) on samples sintered for 10, 30, and 50 hours. Based on the diffraction patterns, slight shifts as well as peak splitting is observed for characteristic peaks of near stoichiometric $\mathrm{Ni}_{2} \mathrm{MnGa}$ phases, probably related to chemical composition inhomogeneity. 
References:

[1] A. Sozinov, et al, Applied Physics Letters 80 (2002), p. 1746.

[2] S. Roth, et al, Trans Tech Publications 52 (2008), p. 29.

[3] M. Chmielus, et al, Nature Materials 8 (2009), p. 865.

[4] M. Caputo, et al, Materials Science and Technology Conference Proceedings (2015), p. 1669.

[5] The use of the EM and AM Facilities within the Center for Excellence in Materials Science and Engineering at Youngstown State University is gratefully acknowledged. This work was supported by NSF, DMR grant 1229129.
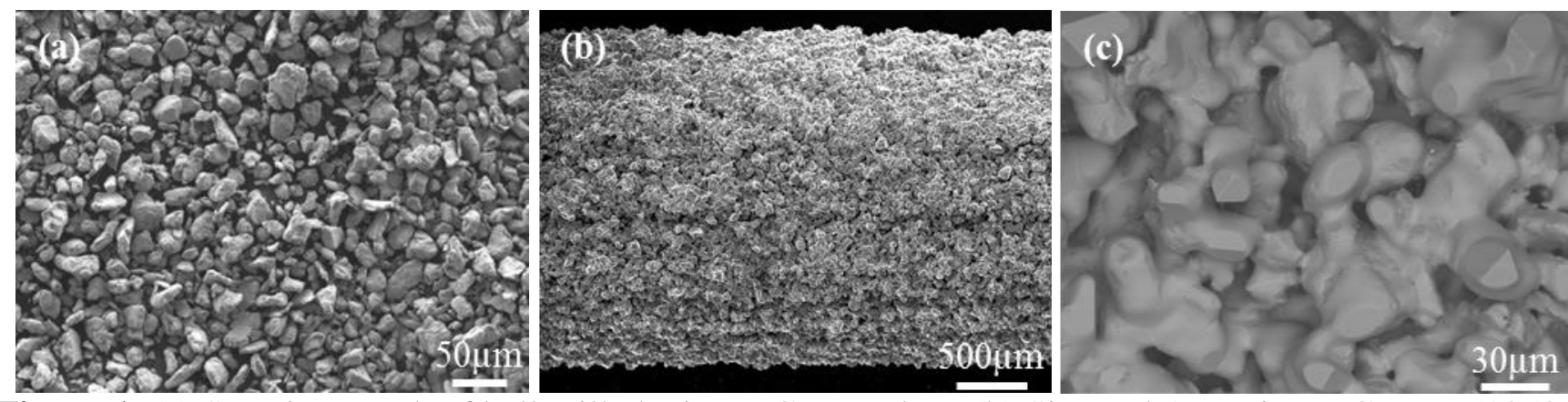

Figure 1. (a) SE micrograph of ball milled Ni-Mn-Ga powders, (b) Sintered AM Ni-Mn-Ga part (1353 $\mathrm{K}, 20$ hours), (c) BSE of sintered Ni-Mn-Ga.
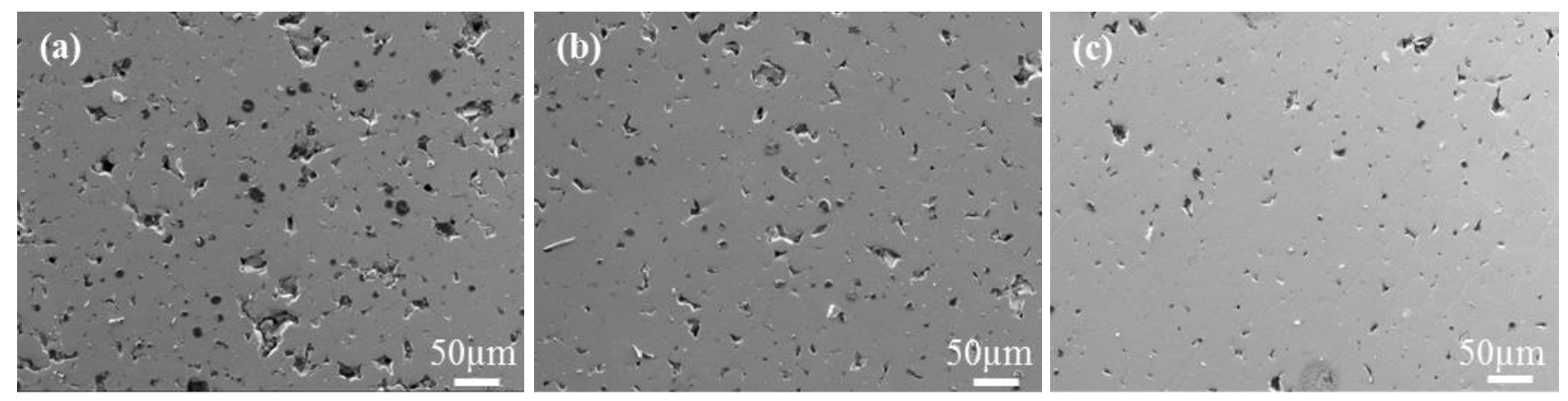

Figure 2. SE micrographs of polished cross-sectioned Ni-Mn-Ga AM parts sintered at (a) 10, (b) 30, and (c) 50 hours.

\begin{tabular}{|c|l|l|l|}
\hline & Ni (at\%) & Mn (at\%) & Ga (at\%) \\
\hline Powders & $51.50 \pm 1.08$ & $30.07 \pm 0.34$ & $18.43 \pm 0.96$ \\
\hline $10 \mathrm{hr}$ & $49.80 \pm 0.43$ & $30.27 \pm 0.40$ & $19.93 \pm 0.78$ \\
\hline $20 \mathrm{hr}$ & $49.45 \pm 0.30$ & $30.10 \pm 0.30$ & $20.45 \pm 0.51$ \\
\hline $30 \mathrm{hr}$ & $49.66 \pm 0.29$ & $30.63 \pm 0.26$ & $19.71 \pm 0.51$ \\
\hline $40 \mathrm{hr}$ & $49.50 \pm 0.28$ & $30.34 \pm 0.24$ & $20.16 \pm 0.47$ \\
\hline $50 \mathrm{hr}$ & $49.83 \pm 0.34$ & $30.29 \pm 0.17$ & $19.88 \pm 0.51$ \\
\hline
\end{tabular}

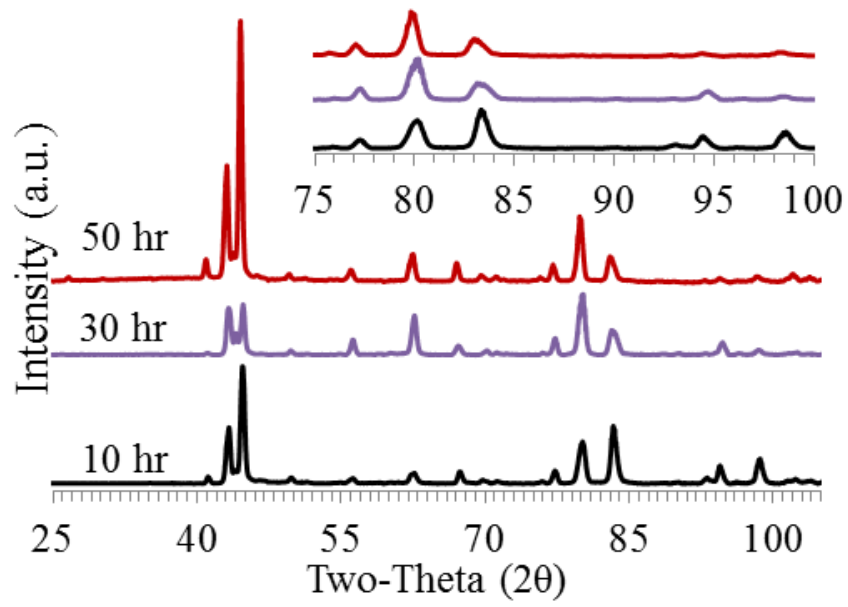

Table 1. Chemical composition of powders and sintered parts. Standard deviation indicated based on 10 measurements.

Figure 3. Diffraction patterns for $\mathrm{Ni}-\mathrm{Mn}-\mathrm{Ga}$ samples sintered at $10,30, \& 50$ hours. 